\title{
A possibilistic approach to diverse-stressor aquatic ecological risk estimation
}

\author{
Sebastian Jooste \\ Institute for Water Quality Studies, Private Bag X131, Pretoria, 0001, South Africa
}

\begin{abstract}
A possibilistic approach to assess the risk of co-occurring stressors in an aquatic ecosystem based on the use of fuzzy sets is illustrated at the hand of a hypothetical case study. There are two aspects of importance: a fuzzy stressor response relationship where the response may have reference to a lower level end-point, and a rule-based inference model relating the occurrence of low-level stressors to a high-level ecological goal such as sustainability. The stressor-response is expressed as a conditional possibility. The possibility and necessity measures of the disjunctive composition of the stressor-response with the possibility distribution of the stressors yield an estimate of the ecological risk. Such a possibilistic approach may well serve as a screening procedure in multiple stressor resource management when only qualitative risk assessments are needed.
\end{abstract}

\section{Introduction}

The South African National Water Act places a premium on water supply for basic human needs and for the sustainable development and use of the aquatic ecosystem. This is reflected in the reserve. The ecological component of the reserve has been defined as that level of quantity and quality necessary to ensure the sustainable development of the water resource (NWA, 1998). The ecological reserve is a water resource management instrument for aquatic ecosystem protection to ensure sustainability in the use and development of the water resource. As a practical management measure, the capacity of the water resource to maintain its sustainability can be discretised into different management classes (MacKay, 1998) corresponding to different levels of risk that the resource may lose its sustainability.

Risk is used here in the sense of the likelihood that a specific undesired event would occur. This likelihood may be expressed in terms of either probability or possibility. In probabilistic risk assessment, it is assumed that this event is crisply defined, i.e. it is possible to decide whether the event has occurred or not. However, the nature and epistemology of the event would determine how likelihood is expressed. Possibility theory offers the option of addressing fuzzy events where the event is perhaps epistemologically vague.

A point of departure in this paper is the recognition that in assessing the risk of the aquatic ecosystem losing its sustainability:

- there are several stressors (such as chemical substances, flow reduction and habitat degradation) that may be present simultaneously and that may result in responses such as loss of sustainability (although the mechanics of these impacts may differ), and

- unambiguous quantitative and possibly even quantitative site specific data may often be lacking.

An argument will be presented for the application of a fuzzy approach to aquatic ecological risk. Two types of ecological risk

푱(012) 808-0374; fax: (012) 808-0338; e-mail: joostes@ dwaf.gov.za Received 22 July 1999; accepted in revised form 23 February 2001. may be defined depending on how the likelihood measure is expressed: a risk based on a possibility measure (referred to as "ecological concern") and a risk based on a necessity measure (related to the possibility measure and referred to as "ecological dread"). These are illustrated by a hypothetical application to water resource classification.

\section{Rationale for a fuzzy approach}

The term "sustainability" is not defined in the NWA. For the purpose of discussion, it is assumed that ecological sustainability refers to the ability of a system to maintain an acceptable level integrity subject to anthropgenic stress. Concepts such as sustainability and integrity may be spatially and temporally scaledependent and the knowledge of the mechanisms underpinning these phenomena is vague (Costanza et al., 1993, De Leo and Levin, 1997). Variability is both a normal and sometimes a necessary ecosystem characteristic tocertain ecosystem processes. "Therefore, in managing ecosystems, the goal should not be to eliminate all forms of disturbance, but rather to maintain processes within limits or ranges of variation that may be considered natural, historic or acceptable" (De Leo and Levin, 1997).

Not only must natural variability be accounted for in the management process, but also uncertainty and, in some cases, vagueness. Definitions of ecosystem integrity varies; e.g. "the maintenance of the community structure and function characteristic of a particular locale or deemed satisfactory to society" (Cairns, 1977) or "the capability of supporting and maintaining a balanced, integrative, adaptive, community of organisms having species composition, diversity, and functional organization comparable to that of natural habitats of the region" (Karr and Dudley, 1981). Terms such as "deemed satisfactory"; "balanced", "comparable" and "natural" in these definitions are, without further qualification, essentially vague and subjective. This means that in terms of the risk assessment under the NWA, the end-point is vague.

In addition, the system boundaries, the response to stressors and the stressors themselves may only be known qualitatively. The functional entities that best reflect the goals of ecosystem management may only be vaguely identifiable. Consequently, in dealing with ecological risk in the context of protective ecosystem management, it would be advantageous to use a paradigm that is 
adapted to address both uncertainty and vagueness. This could be accomplished by using the framework of possibility theory (as opposed to probability theory), which is based on the use of fuzzy logic (as opposed to 'crisp' logic).

\section{Probabilistic vs. fuzzy risk}

Risk is a way of expressing the uncertainty of observing some event (Suter, 1993). The use of risk techniques in decision-making is largely motivated by the variability and uncertainty observed in dealing with ecosystems and has been used extensively in a number of countries (e.g. USEPA, 1996; Pederson, et al., 1995). Probabilistic risk assessment depends crucially on the ability to derive some expression of probability for a stressor variable. Conventionally, imperfect information has been dealt with either by probability or by interval analysis.

Probability theory has, over a period of 200 years, developed a calculus to deal with stochasticity. A problem with probability theory in ecological risk assessment may relate to the interpretation of what is really represented by probability (Dubois and Prade, 1988). The frequentist approach sees probability as the limiting frequency of observed, clearly defined events. The first major obstacle in assigning probability distributions for ecological variables is the lack of enough system-specific information to estimate these limiting frequencies. The alternative Bayesian approach circumvents the frequentist dilemma by using probability as a descriptor of the state of knowledge about an event or proposition (Jaynes, 1996) and is often much better suited to generating the necessary distribution data.

The second (and possibly more critical) problem facing ecological risk assessment and risk management is the difficulty in defining the system uniquely at an operational level. The boundaries of ecosystems, communities and even populations, for example, are notoriously vague. This complicates the use of both frequentist and Bayesian statistics, which deal with such vagueness with difficulty. Mathematically, this vagueness, superimposed on the complexity of ecosystems, the elements of which may exhibit stochastic behaviour, results in analyses that become intractable to conventional mathematics. The resulting ecosystem models exist largely as lexical system descriptions. In analyzing a complex multidimensional system, a state could be reached where, even if uncertainty and variability could be quantified, the results would be difficult to interpret (Dubois and Prade, 1988). As the complexity of the system or model of a system increases, a point could be reached where "our ability to make precise and yet significant statements about its behaviour diminishes until a threshold is reached beyond which precision (or relevance) becomes almost mutually exclusive characteristics" (Zadeh, 1973)

Working with incomplete data, ecologists may have to deal largely with judgement, which by its nature has at least an element (if not consisting entirely) of subjective opinion. Possibility theory in contrast to probability theory, "offers a model for the quantification of judgement which allows a canonical generalisation of interval analysis" (Dubois and Prade, 1988) which has been used in the analysis of uncertainty in the physical sciences.

Risk estimation in ecosystems has been shown to be influenced by both uncertainty and variability (e.g. Frey, 1993, Frey and Rhodes, 1999), which argues for a probabilistic rather than a deterministic approach in assessment. The concept of risk contains the elements of:

- value ("what is being threatened"),

- extent ("how badly"),

- the likelihood of a) and b), and

- assessment ("what does it mean").

\section{Applying possibility theory to assessment of ecological reserve-related risk}

For discrete events $\omega$ with a possibility distribution $\pi(\omega)$, the possibility measure $\operatorname{Poss}(\mathrm{A})$ and the necessity measure $\mathrm{Nec}(\mathrm{A})$ are defined by Eq.1.

$$
\begin{aligned}
& \operatorname{Poss}(A)=\sup \{\pi(\omega) \mid \omega \in A\} \\
& \operatorname{Nec}(A)=\inf \{1-\pi(\omega) \mid \omega \notin A\}
\end{aligned}
$$

Some of the differences between probability measures and possibility or necessity measures are:

- The probability of the sure event is assigned the value 1 . For a number of events, the cumulative probability of all possible events is assigned the value 1 . A possibility of 1 , however, does not imply that the event is sure, only that it is entirely possible.

- The knowledge of the probability of an event completely determines the knowledge of the contrary event. Knowledge of the possibility or necessity of an event is less strongly linked to the knowledge of the contrary event. To establish the certainty of an event, it is necessary to know both the possibility and the necessity of the event.

- Probability deals with precise but differentiated items of information. Possibility reflects imprecise but coherent items.

- A central requirement in probability theory is the additivity of the probability of independent, mutually exclusive (disjoint) events. This requirement, generally, does not hold for fuzzy likelihood measures.

These characteristics of possibility theory make possibility measures well-suited to reasoning in an uncertain environment where it is often desirable not to set the relationship between the evidence one has for an event (degree of necessity) and the evidence that weighs against it (1-degree of possibility) too rigidly. In addition, it might be prudent to consider whether one's knowledge that an event (such as loss of sustainability) might occur, also defines the possibility that the event might not occur. In other words, does one's knowledge of the ecosystem allow for the law of the excluded middle of Aristotelian ('crisp') logic?

Variability: an inherent and practically irreducible characteristic of a biotic system, stemming from the innate stochasticity underlying processes in the ecosystem.

Uncertainty: epistemic of the observer stemming from imperfect information, due to limitations in observation, modelling or interpretation of system-related data, for example.

Vagueness (or fuzziness): a lack of clarity in the definition of the set of values attached to the object.

Ambiguity: largely associated with language, where the definition of the object is vague or refers to several different reference sets simultaneously. 


\section{The regulatory end-point $E$}

In an ecological risk assessment implicit in the classification in terms of the reserve, the "regulatory" undesired event, $E$, is defined by the NWA as "loss of sustainability". This is a fuzzy event in the light of the foregoing. The management classes in the NWA correspond to differences in the likelihood of this fuzzy event occurring.

This definition of $E$ implies that it is a dichotomous characteristic of the system; anything less than full sustainability means unsustainability. It does not mean that important related characteristics such as resilience and integrity need to be dichotomous as well. There might be levels of resilience and integrity less than $100 \%$ that still result in sustainability. $E$ may be epistemologically vague, in that the knowledge of what constitutes $E$ (or $\neg E$ i.e. "not $E$ ") may be imperfect. An assessment of the "likelihood of $E$ " may be a reflection of the epistemology of the values of the parameters defining the critical point defining $E$. Consequently, the evidence one has that a certain set of parameter values corresponds to $E$ and the evidence that it corresponds to $\neg E$ might not be complementary in the sense that one's knowledge of $E$ occurring does not define one's knowledge of $E$ not occurring. There might, therefore, be a set of parameter values for which it is not possible to make a clear assessment of either the likelihood of $E$ or the likelihood of $\neg E$. The "likelihood of $E$ " is interpreted as the degree to which the observed situation corresponds to $E$.

\section{Ecological concern and dread}

The likelihood aspect of risk can be expressed in terms of possibility theoretical concepts. Poss (E) could be used to express the possibility that effect $E$ would occur. This does not carry the same weight as the probability of $E, \mathrm{P}(E)$. It is always true that $N e c(E)$ $\leq \mathrm{P}(E) \leq$ Poss $(E)$. This means that Poss $(E)$ expresses an epistemic possibility that $E$ could occur and therefore, Poss $(E)$ expresses a weaker claim than $\mathrm{P}(E)$. More appropriately, Poss $(E)$ might designate the degree of "ecological concern".

On the other hand, $\mathrm{Nec}(E)$ expresses the cumulative evidence of the necessity that $E$ must occur. This is a much stronger claim that $\mathrm{P}(E)$ and may appropriately be expressed as the degree of "ecological dread". Both ecological concern and ecological dread express the accumulated evidence about the likelihood that the undesired event $\mathrm{E}$ will occur.

There are three aspects to the assessment of ecological risk in the aquatic environment that are important in the context of the reserve:

- The estimation of the aggregate likelihood of $\operatorname{Poss}(E)$ or $\operatorname{Nec}(E)$ when diverse stressors occur together,

- The confidence in Poss $(E)$ or Nec $(E)$ on projecting $E$ from other available data and

- The formulation of the relationship between Poss $(E)$ or Nec $(E)$ and the stressor value.

\section{Aggregating diverse stressors}

There are a number of different stressors that could result in loss of sustainability. Assume, for example, that flow deficiency (i.e. degree to which the flow is less than that expected in the natural hydrological cycle), toxic substances and habitat degradation are typical stressors in a system being assessed. In order for $E$ to occur, it is assumed that:
- An environmental variable $X$ with value $x$, only becomes a stressor if it can result in $E$, i.e. in the present context, stress is not defined if a variable is within its natural range of variability. Furthermore, there exists a critical value $x_{0}$ at which $E$ occurs. Our knowledge (rather than the inherent nature) of $E$ as well as $x_{0}$ make both fuzzy quantities. The likelihood of E occurring (both Poss $(E)$ or $N e c(E)$ ) is a function of $x$. The stress $E_{X}$, which is used here in the sense of the extent of the effect $E$ being produced as a result of stressor $X$, depends on a fuzzy causal relationship $E \mid X$ and an occurrence of stressor $X$, where the $X$ is a fuzzy set of stressor values which correspond to $x_{0}$ and which is defined in terms of the degree to which a value $x$ corresponds to $x_{0}: X=\left\{x \mid \mu_{X}(x)=\pi\left(x=x_{0}\right)\right\}$.

- Any of the stressors could result in $E$, irrespective of whether they occur alone or together. The ecological concern would refer to the possibility that any of the stressors (and by implication the resultant stresses) occur. The ecological dread would refer to the necessity that all the stressors occur together (in which case there is no doubt in the assessor's mind that $\mathrm{E}$ is likely to occur).

- Generally, it would not be known (at least at the outset) whether there is an additive, supra-additive ("synergistic") or infraadditive ("antagonistic") interaction among stressors. The way in which this is approached is largely a matter of assumption until further evidence is produced. The assumption will be reflected in the risk aggregation operators (t-norm and tconorm in Eq. (3) below).

For the stressors noted above, these assumptions could be interpreted as:

- There exists a value of flow, $q_{0}$, in a given river section, for example, which will result in loss of sustainability if this flow is maintained for a sufficient period. Although the exact value is unknown, flow requirement studies (e.g. King and Louw, 1998) may yield some idea of what it might be. The flowrelated concern and dread for any specific value of flow, $q$, under discussion, can be estimated from Eq. (2a):

$$
\begin{aligned}
& \operatorname{Poss}\left(E_{Q}\right)=\operatorname{Poss}((E \mid Q) \wedge Q)=t-\operatorname{norm}\{\operatorname{Poss}(E \mid Q), \operatorname{Poss}(Q)\} \\
& \operatorname{Nec}\left(E_{Q}\right)=t-\operatorname{norm}\{\operatorname{Nec}(E \mid Q), \operatorname{Nec}(Q)\}
\end{aligned}
$$

- There exists a critical value of toxic substance concentration, $t_{0}$, (as toxicity units) such that for any specific value $t$ the toxicsrelated concern and dread would be given by Eq. (2b).

$$
\begin{aligned}
& \operatorname{Poss}\left(E_{T}\right)=\operatorname{Poss}((E \mid T) \wedge T)=t-\operatorname{norm}\{\operatorname{Poss}(E \mid T), \operatorname{poss}(T)\} \\
& \operatorname{Nec}\left(E_{T}\right)=t-\operatorname{norm}\{\operatorname{Nec}(E \mid T), \operatorname{Nec}(T)\}
\end{aligned}
$$

- Analogous to the above, the fuzzy critical habitat degradation value $H$ is assessed by expert opinion so that for any specific level of habitat degradation, $h$, the habitat-related concern and dread will be given by Eq. (2c).

$$
\begin{aligned}
& \operatorname{Poss}\left(E_{H}\right)=\operatorname{Poss}\left(\left(E \mid h_{0}\right) \wedge H\right)=t-\operatorname{norm}\{\operatorname{Poss}(E \mid H), \operatorname{Poss}(H)\} \\
& \operatorname{Nec}\left(E_{H}\right)=t-\operatorname{norm}\{\operatorname{Nec}(E \mid H), \operatorname{Nec}(H)\}
\end{aligned}
$$

The fuzzy set $X$ is normalised since by assumption a stressor is only defined as such if there is at least one value of $x$ such that $\mu_{X}(x)=1$, i.e. there is at least one value for which $E$ is entirely possible. Hence, the equivalence of the membership function values with the possibility of $\mathrm{X}$.

A further result of the assumptions above is that the ecological concern $\rho_{c}$ and ecological dread $\rho_{d}$ is expressed in Eq. (3): 


\begin{tabular}{|l|l|l|l|l|}
\hline \multicolumn{5}{|c|}{ TABLE 1} \\
Some possible t-norms and -conorms (Kruse et al., 1994) for use as aggregation operators on quantities \\
a and $\boldsymbol{b}$ in assessing $\boldsymbol{\rho}_{c}$ and $\boldsymbol{\rho}_{\boldsymbol{d}^{*}}$
\end{tabular}

$$
\begin{aligned}
\rho_{c}=\operatorname{Poss}(E) & =\operatorname{Poss}\left(E_{Q} \vee E_{T} \vee E_{H}\right)=t-\operatorname{conorm}\left\{\operatorname{Poss}\left(E_{Q}\right), \operatorname{Poss}\left(E_{T}\right), \operatorname{Poss}\left(E_{H}\right)\right\} \\
& =\min \left\{\sum_{X \in\{Q, T, H\}} \operatorname{Poss}\left(E_{X}\right), 1\right\} \\
\rho_{d}=\operatorname{Nec}(E) & =\operatorname{Nec}\left(E_{Q} \wedge E_{T} \wedge E_{H}\right)=t-\operatorname{norm}\left\{\operatorname{Nec}\left(E_{Q}\right), \operatorname{Nec}\left(E_{T}\right), \operatorname{Nec}\left(E_{H}\right)\right\} \\
& =\max \left\{0, \sum_{x \in\{Q, T, H\}} \operatorname{Nec}\left(E_{X}\right)\right\}
\end{aligned}
$$

The implication is that if $\rho_{c}=0$ then $E$ is considered impossible (inasmuch as our knowledge base allows for that) and $\rho_{d}=0$ by definition. If $\rho_{c}=1$, then $E$ is considered entirely possible (of course not necessarily entirely probable) and $\rho_{d}$ may be $\geq 0$, which means that not only is $E$ possible, but it may also necessarily occur. If $0<\rho_{c}<1$, then $E$ is possible to the extent $\rho_{c}$ but $\rho_{d}=0$ (if an event is not entirely possible it cannot be at all necessary).

The choice of $t$-norm and t-conorm in Eq. (3) for the stress aggregation needs to take cognisance of the knowledge about the interaction among stresses. For toxic substances, true synergism among the substances appears to be rare (Hermens et al., 1984a; 1984b; Calamari and Vighi, 1992) although it has been reported (Broderius and Kahl, 1985). Additivity of toxicity occurs more often than true synergism or supra-additivity. For other stressors, effects such as additivity have not been reported on if they do exist. Even less so has synergism among diverse stressors been reported on.

It is likely that additivity of effect among diverse stressors reflects the worst case, while additivity may also be possible. Some of the possible t-norms and-conorms that could be used in aggregating fuzzy risks are listed in Table 1.

For the aggregation of concern and dread (Eq. (3)) the Lukasiewicz aggregation with the implied additivity of stresses appears to the most conservative option. For the aggregation of risk components (Eqs. (2a) to (2c)), exposure and effect may be seen as contributing independently to the likelihood of effect, and consequently, the min-max aggregation would be more suitable.

\section{End-point projection}

The regulatory end-point $E$, which is at ecosystem scale, is unlikely to have data at the correct spatial and temporal scale from which it can be derived. It is more likely that, on a case-specific basis, phenomena at smaller spatial and temporal scales will be used to infer the occurrence of $E$. Lower level phenomena such as the disappearance of key species, loss of integrity, mortality of selected species are more likely to be used to infer $E$.

For example, assume that a toxic substance is introduced into a river system. From toxicity assessment it might be established that if the concentration of the toxic substance is $x$ then the cumulative probability of an individual in a population of the test species $Z$ will die, is $y$, with confidence interval $\left(y_{1}, y_{2}\right)$. The toxicity concern , $\operatorname{Poss}\left(E_{T}\right)$, and dread, $\operatorname{Nec}\left(E_{T}\right)$, must be estimated from these data. In order to do this, it is necessary to follow some conceptual inference model such as Eq. (4)

\section{Rule base (R):}

IF concentration IS $x$ THEN an individual of species Z IS dead $\left(\right.$ Possibility $\left.=y_{l}\right)$

IF an individual of species Z IS dead THEN the population of Z IS lost $($ Possibility $=\alpha)$

IF the population of Z IS lost THEN a key species IS lost $($ Possibility $=\beta)$

IF a key species IS lost THEN integrity of the ecosystem IS irreversibly compromised (Possibility $=\gamma$ )

IF integrity of the ecosystem IS irreversibly compromised THEN sustainability IS lost (Possibility $=\delta$ )

Observation $(X)$ : The concentration IS $\mathrm{x}($ Possibility $=\varepsilon)$

An analogous rule base can be formulated for $\mathrm{N}\left(E_{T}\right)$. The value of Poss $\left(E_{T}\right)$ derives from the conjunction $R \wedge P$. This value will be a function of $y_{l}, \alpha, \beta, \gamma, \delta$ and $\varepsilon$. In its simplest form Poss $\left(E_{T}\right)$ $\leq \min \left\{y_{l}, \alpha, \beta, \gamma, \delta, \varepsilon\right\}$. (For $N e c\left(E_{T}\right)$ the inequality will be replaced by an equality.) This would support the notion that the possibility that toxics cause a loss of sustainability can be no stronger than the weakest inferential link. Since specific data for their assessment is usually lacking, the values for $\alpha, \beta, \gamma, \delta$ and $\varepsilon$ may conservatively be set equal to 1 . The assumption should not simply be made that the confidence in the lower level phenomenon is equal to that of $E$ (Suter, 1993; 1995).

\section{Stressor-response relationships}

A crucial component of the individual stressor concern (or dread) assessment is the conditional term Poss $\left(E \mid x_{0}\right)$ or Nec $\left(E \mid x_{0}\right)$. These terms are essentially the output of the effect assessment phase of an ecological risk assessment in the context where an end-point is fixed. It summarises the knowledge about the expectation of effect of the stressor on the system being assessed and answers the implied question: "What if the system is being exposed to the stressor"? In the present context, both $E$ and $x_{0}$ are fuzzy entities and, hence, the condition term represents a fuzzy relationship, $R_{X}$. $R_{X}$ is the formalised knowledge base on the relationship between the likelihood of $E$ and $x$. The likelihood of individual stresses is derived from $R_{X}$ and an observation $X$ by Eq. (5). An expression for $\operatorname{Nec}\left(E_{X}\right)$ can be similarly derived from Eq. (1).

$$
\begin{aligned}
& \operatorname{Poss}\left(E_{X}\right)=R_{X}{ }^{i} \circ X=\sup _{x}\left\{t-\operatorname{norm}\left\{\mu_{X}(x), R_{X}(E, x)\right\}\right\} \\
& =\sup _{x}\left\{\min \left\{\mu_{X}(x), R_{X}(E, x)\right\}\right\}
\end{aligned}
$$


The relationship $R_{X}$ derives from a rule-base of the kind "If $X=x$ then $E=\varepsilon$ " where the truth-value of $X=x$ is $\mu_{X}(x)$ and that of $E=\varepsilon$ is $\mu_{E}(\varepsilon)$. This can then be formulated as " $\mu_{X}(x) \rightarrow \mu_{E}^{\prime}(\varepsilon)$ ". Using the max-min implication (Table 1) Eq. (5) becomes Eq. (6).

$$
\operatorname{Poss}\left(E_{X}\right)=\sup \left\{\min \left\{\mu_{X}(x), \min _{\varepsilon}\left\{1-\mu_{X}(x)+\mu_{E}(\varepsilon), 1\right\}\right\}\right\}(6)
$$

Evaluating $R_{X}$ now becomes the problem of evaluating the relationship $\mu_{X}(x) \rightarrow \mu_{E}(\varepsilon)$, or "IF $\mu_{X}(x)$ THEN $\mu_{E}(\varepsilon)$ ". There are two distinct ways to generate this assessment:

- Cause to effect: Given a stressor value $\mathrm{x}$, to what extent will its impact comply to the description $E$ (i.e. $x \rightarrow E$ ) and

- Effect to cause: Given a level of effect $\varepsilon$, what are the levels of $\mathrm{x}$ that correspond to $\varepsilon$ (i.e. $E \rightarrow x$ ).

In general, this need not be a mathematical-functional relationship. If the best knowledge available is in the form of fuzzy "rules" such as those in Eq. (4), then the stressor-response relationship (SRR) is at best a fuzzy mapping of the stressor value domain to the response likelihood domain.

\section{Hypothetical case study}

In an ecological risk assessment study, it is agreed that there are three major stressors in a catchment, i.e., unidentified toxic substances, deviation from expected flow and physical habitat degradation. There are three types of information that is required from expert input:

- Definition of the SRR from a) the lowest stressor value where effect $E$ may be expected to be discernable $\left(x_{11}\right)$, b) the lowest stressor value where $E$ may be entirely possible $\left(x_{12}\right)$, c) the highest level where E may be discernable $\left(x_{21}\right)$ and d) the highest level where $E$ is entirely possible $\left(x_{22}\right)$.

- The epistemological confidence on projecting from the observable response to the regulatory end-point $(\alpha, \beta, \gamma, \delta$ and $\varepsilon)$.

- The likelihood of the occurrence of the stressor. $\left(\mu_{X}(x)\right)$

\section{Fuzzification of concern and dread}

Consider a situation in a river system where the critical effect, $E$, being assessed is "loss of sustainability". Due to the epistemic uncertainty relating to mechanisms, thresholds, subjectivity in assessments, etc. in a river system, the risk of $E$ (expressed here as the possibility of $E$ ) is described in terms of categories rather than numerical terms. For example, the level of risk may be assessed as belonging to a class $K$ such that the set $K=$ \{Insignificant, Low, Marginal, Significant and High $\}$ as shown in Fig. 1.

These classes are vague since their boundaries may be a matter of interpretation. An effect possibility of 0.2 might be described as being 'low' or 'marginal' to some extent. Consequently, the classes are modelled as fuzzy sets. These same 'fuzzification' parameters might also be used in describing the concern and dread levels since they deal with the same type of possibilistic measures.

The definition of individual stressor effect possibility (Eq. (6)), as well as the aggregated concern values (Eq. (3)), ensure that at least one of the fuzzy sets will have a membership value of 1 . This means that it will be possible to describe the concern level in a river or stream in terms of at least one of the classes. However, it may be possible that more than one class has a membership of 1 , in which case the worst class that has a membership of 1 will logically be class descriptor for the river situation.

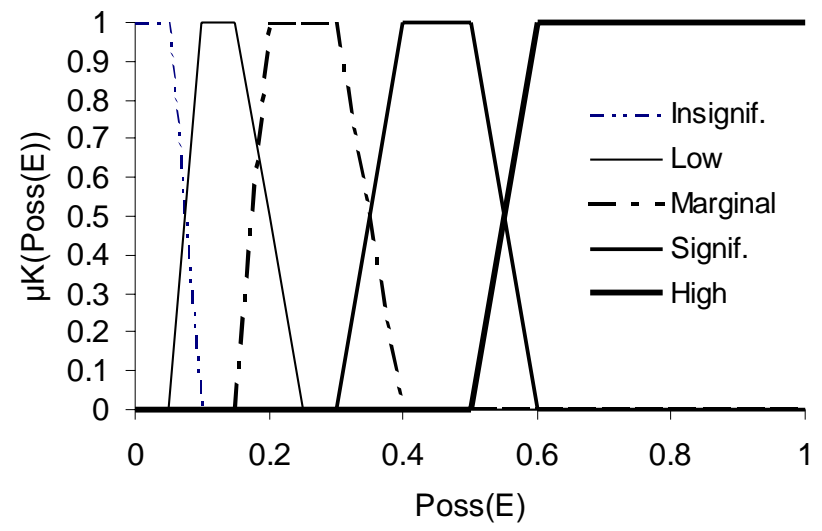

Figure 1

The parameters for describing the possibility of $\mathrm{E}$ in terms of the set $\mathrm{K}$ of fuzzy labels. The fuzzy set is defined by the degree to which the possibility of effect, Poss(E), corresponds to the descriptor $\mathrm{K}$.

\section{Toxic stress}

The toxicity stress is determined by toxicity bioassessment studies without specifically identifying the toxic components and is expressed as toxicity units. A toxicity unit is defined as 100 divided by a benchmark effect concentration expressed as a percentage of the effluent. The data are derived from single species toxicity tests and projections of effect to population level (e.g. Caswell, 1989). The no-observable-effect concentration (NOEC) is taken to be at $10 \%$ of the EC50.

In-stream objectives of 0.3 TUa and 1 TUa have been suggested as levels where no critical effects should be observed (USEPA, 1991, Tonkes and Balthus, 1997) and these values are used for $x_{11}$ and $x_{21}$ respectively. It is assumed that at double the EC50, sustainability might be lost if predation pressure is high while, even under the best circumstances, sustainability is in jeopardy if $99 \%$ (corresponding perhaps to 3 times the EC50) of a population dies. These values are used for $x_{12}$ and $x_{22}$ respectively (Fig. 2(a)). The possibility distribution for $x$ is assumed to be a triangular distribution such that $\mu_{X}(x)=0$ corresponds to the $5^{\text {th }}$ and $95^{\text {th }}$ percentile values while $\mu_{X}(x)=1$ corresponds to the median value. The values of $\alpha$, $\beta, \gamma, \delta$ and $\varepsilon$ in Eq. (4) are all assumed to be 1 .

\section{Flow stress}

The flow stress, $q$, is assumed to be due to the reduction of the expected flow in stream. The value of $q=0$ when the stream flow is very similar to pristine flow while $q=1$ corresponds to critical disruption of stream flow. The values for the mapping parameters are entirely hypothetical (Fig. 2(b)).

\section{Habitat stress}

The habitat degradation is assessed by a river ecologist and expressed as a percentage deviation from what is expected to be pristine. The values for the mapping parameters are entirely hypothetical (Fig. 2(c)). The fuzzy relationships were assumed to show a triangular distribution such that for any stressor level, the effect is given by a triangular distribution with its least likely values given by $y_{1}$ and $y_{2}$ (see Appendix) and its most likely value by $y_{m}$. 
TABLE 2

The scenarios in which the ecological risk assessment is evaluated.

\begin{tabular}{|c|c|c|c|}
\hline Scenario & Toxic substance status & Flow status & Habitat status \\
\hline 1 & $\begin{array}{l}\text { The levels are practically pristine. } \\
\text { Discharges are mostly assimilated }\end{array}$ & $\begin{array}{l}\text { Very little abstraction or water loss } \\
\text { is evident. Sporadic abstraction has } \\
\text { a minor impact. }\end{array}$ & $\begin{array}{l}\text { Practically pristine. Only minor } \\
\text { modifications }(10 \%) \text { are found. }\end{array}$ \\
\hline 2 & $\begin{array}{l}\text { Substantial discharges exist. With a } \\
\text { very low frequency up to } 5 \mathrm{TUa} \text { is } \\
\text { found while there is usually some } \\
\text { chronic toxicity detected ( } 0.1 \mathrm{TUa}) \text {. } \\
\text { Values of } 1 \text { TUa is found commonly. }\end{array}$ & $\begin{array}{l}\text { Extensive abstraction takes place at } \\
\text { times resulting stressor levels within } \\
20 \% \text { of critical levels. On rare } \\
\text { occasions the flow is practically } \\
\text { pristine, but mostly the flow is } \\
\text { within } 50 \% \text { of pristine. }\end{array}$ & $\begin{array}{l}\text { There is almost no pristine habitat } \\
\text { left with some areas being largely } \\
\text { modified (about } 75 \% \text { ) while most } \\
\text { of the stream has about } 50 \% \\
\text { suitable habitat left. }\end{array}$ \\
\hline 3 & $\begin{array}{l}\text { Rigid control on point sources is } \\
\text { instituted but on rare occasions } \\
1 \mathrm{TUa} \text { is still found, but mostly } \\
\text { toxicity is around } 0.3 \mathrm{TU} \text { a or even as } \\
\text { low as no detectable toxicity. }\end{array}$ & $\begin{array}{l}\text { Some control on abstraction is } \\
\text { possible and flows within } 20 \% \text { of } \\
\text { expected can often be achieved. } \\
\text { However, on rare occasions up to } \\
80 \% \text { of the pristine flow is abstracted. }\end{array}$ & $\begin{array}{l}\text { Some habitat remediation could be } \\
\text { effected so that most of the river } \\
\text { now has } 25 \% \text { loss of the pristine } \\
\text { habitat while the worst case has } \\
\text { only about } 50 \% \text {. }\end{array}$ \\
\hline 4 & $\begin{array}{l}\text { Toxicity is managed to be around } \\
0.55 \text { TUa most of the time while } \\
\text { excursions up to } 1.1 \text { are rarely found. }\end{array}$ & Same as in 1. & Same as in 1. \\
\hline
\end{tabular}
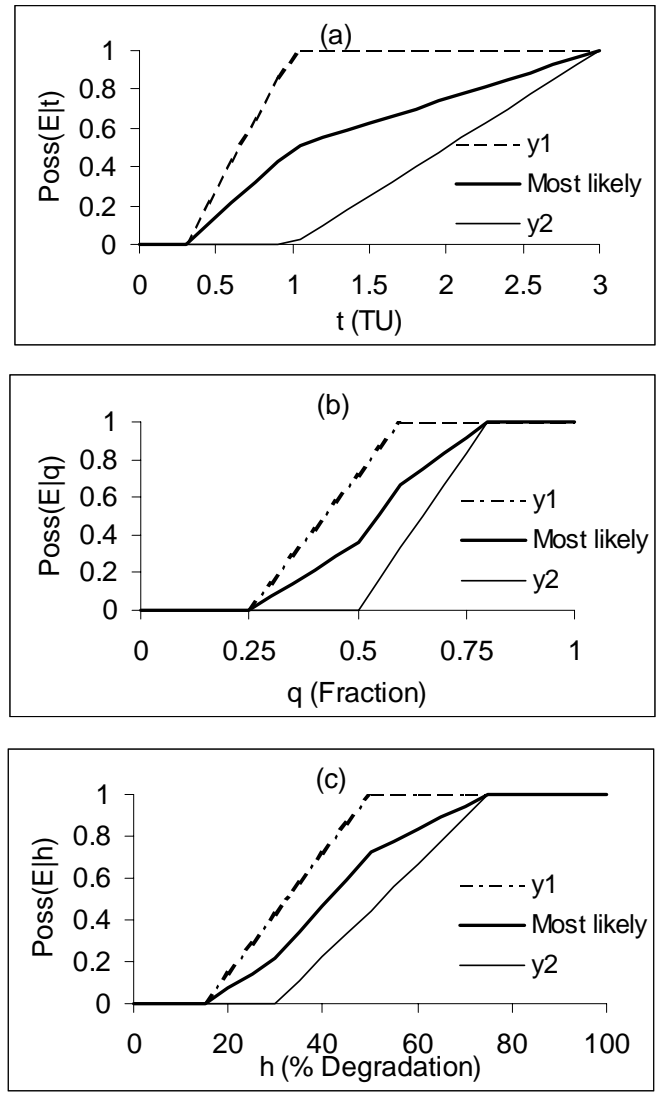

Figure 2

The fuzzy mapping representing the SRR's for the stressors in this study: (a) SRR for toxicity stress, (b) SRR for flow stress and (c) SRR for habitat stress.
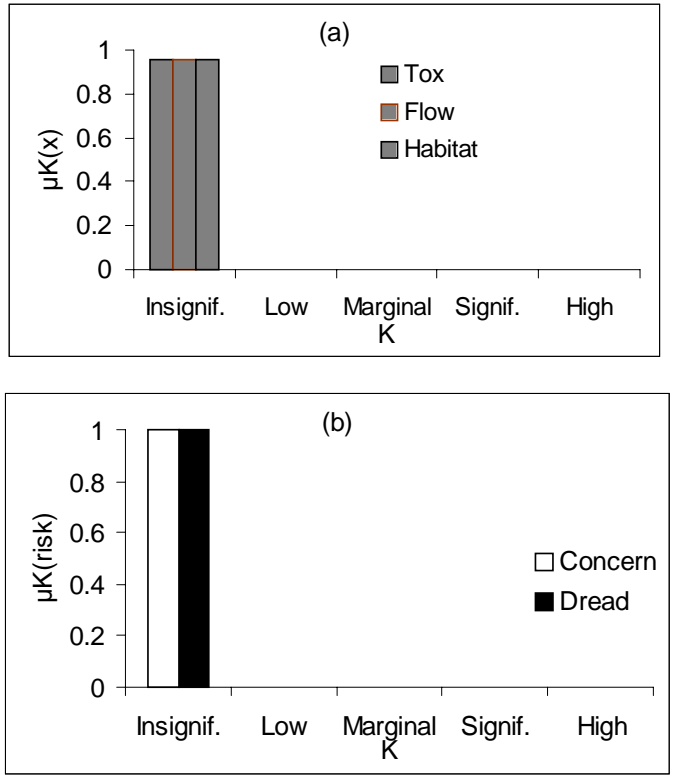

Figure 3

The classification of (a) the stressor specific possibility of effect in terms of fuzzy set membership to the class K (see Fig. 1) and (b) the concern and dread for Scenario 1 (Table 2).

\section{Methodology}

Eqs. (1) to (3), (5) and (6) as well as those in the Appendix were solved using an Excel97 spreadsheet under Windows 95.

The use of ecological concern and ecological dread was investigated by considering its value in four scenarios as described in Table 2.

The narrative description of scenario 1 in Table 2 yields 

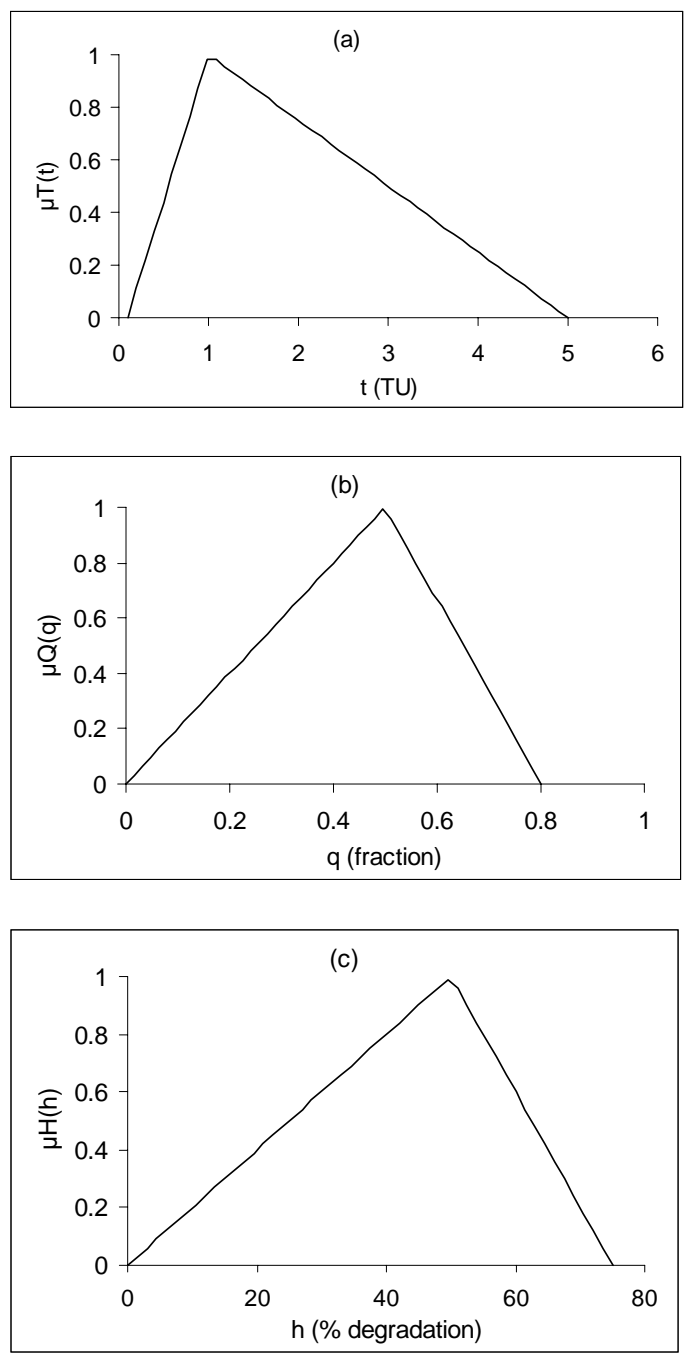

Figure 4

The stressor possibility distributions for (a) toxics-,

(b) flow- and (c) habitat-related stress (expressed as $\left.\mu_{\mathrm{x}}(\mathrm{x})\right)$ derived from the descriptive data for Scenario 2 in Table 2

stressor possibility distributions that are triangular with vertex at $(0,0)$. The stressor possibility distributions for scenario's 2 and 3 are shown in Figs. 4 and 6 while the SRR's are shown in Fig. 2.

\section{Results and discussion}

The individual stressor risks are shown in Figs. 3, 5, 7 and 8.

Scenarios 1 to 3 were chosen to represent a pristine, a heavily utilised and a reasonably managed system respectively. The pristine system, not surprisingly, yielded an assessment of insignificant risk for each individual stressor (Fig. 3(a)). Consequently, both the concern and dread (Fig. 3(b)) are 'insignificant' as would be expected.

In the case of the heavily utilised system (Fig. 5) the individual stressor risk values are either 'significant' (toxics and flow) or 'high' (habitat), considering the maximum membership values. The aggregation method used here results in a concern membership value of 1 to all classes. Since the worst class will reasonably dominate, it could be said that the concern level is 'high'. In this case the dread value is used to distinguish between the classes, so
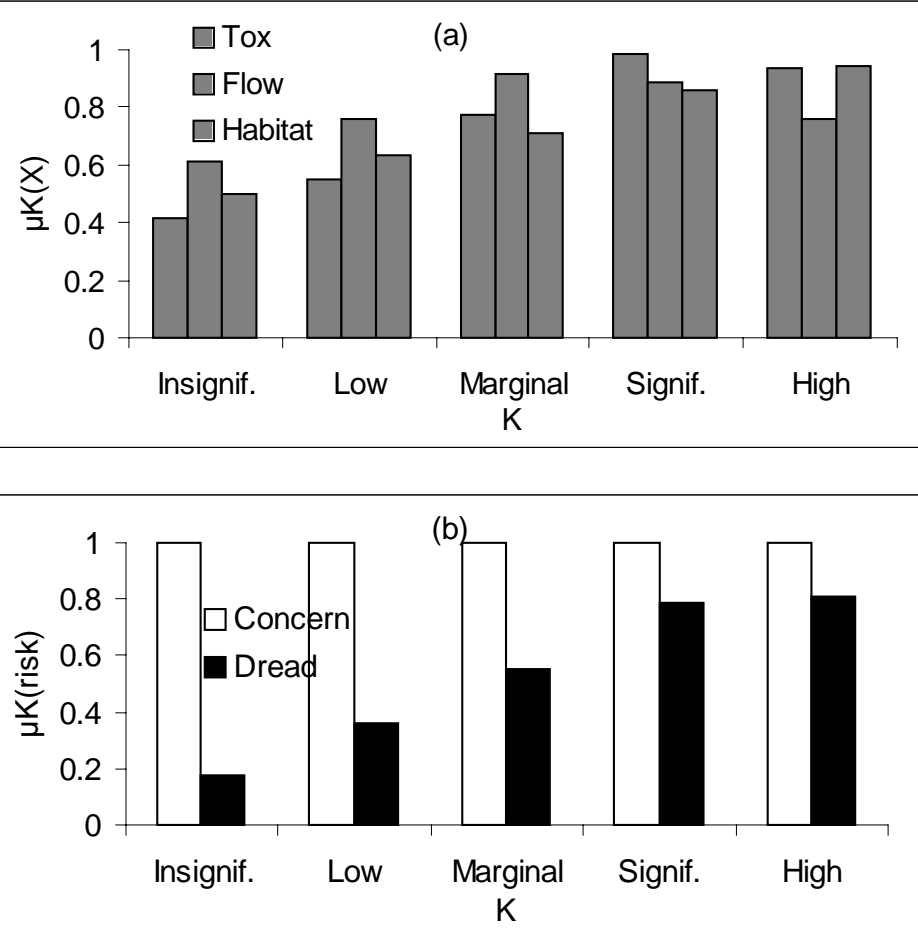

Figure 5

The classification in terms of class K (Fig. 1) of (a) stressor specific effect possibility (Poss(E)) and (b) concern and dread for Scenario 2

that a dread class of 'high' could be allocated.

An analysis of the stressor risk contributions in Scenario 2 shows that all the stressors need attenuation. It is assumed that in the managed system (Scenario 3) it is possible to manage the discharge of toxics as well as abstractions to a reasonable extent while stream habitat remediation is less successful (Fig. 6). The results (Fig. 7) indicate that although toxic and flow risk are now largely 'insignificant' and habitat risk is 'low', on aggregate the concern level is still no better than 'high'. The dread value though has become ' 'insignificant', demonstrating that progress had been made in improving the situation.

Scenario 4 (Fig. 8) was used to illustrate a possible use of concern and dread assessment in assessing the change in criteria (in this case the example of toxicity management criteria). It was now assumed that both habitat and flow risk were insignificant. By systematically changing of the most likely value and the upper limit value in the toxicity possibility distribution, it was attempted to find a parameter set that would be on the verge of changing the concern assessment from 'insignificant' to 'low'. This parameter set is reflected in Table 2. This is in spite of the toxic effect possibility being 'low' or even 'marginal'.

The interpretation of Scenario 4 is that if there are no other stressors that could significantly contribute to the ecological risk, then the parameter values for this scenario will be the maximum allowable to maintain 'insignificant' concern and dread levels.

It has been assumed that risk objectives for the river have been set. This is generally not true for South African rivers. The parameters (i.e. the $\operatorname{Poss}(\mathrm{E})$ values defining the fuzzy set trapezium in Fig.1) used for classifying response possibility are critical. In this hypothetical study the fuzzification as depicted in Fig. 1was simply assumed. No formal procedure was put forward to derive rational values for these parameters and this aspect needs more 

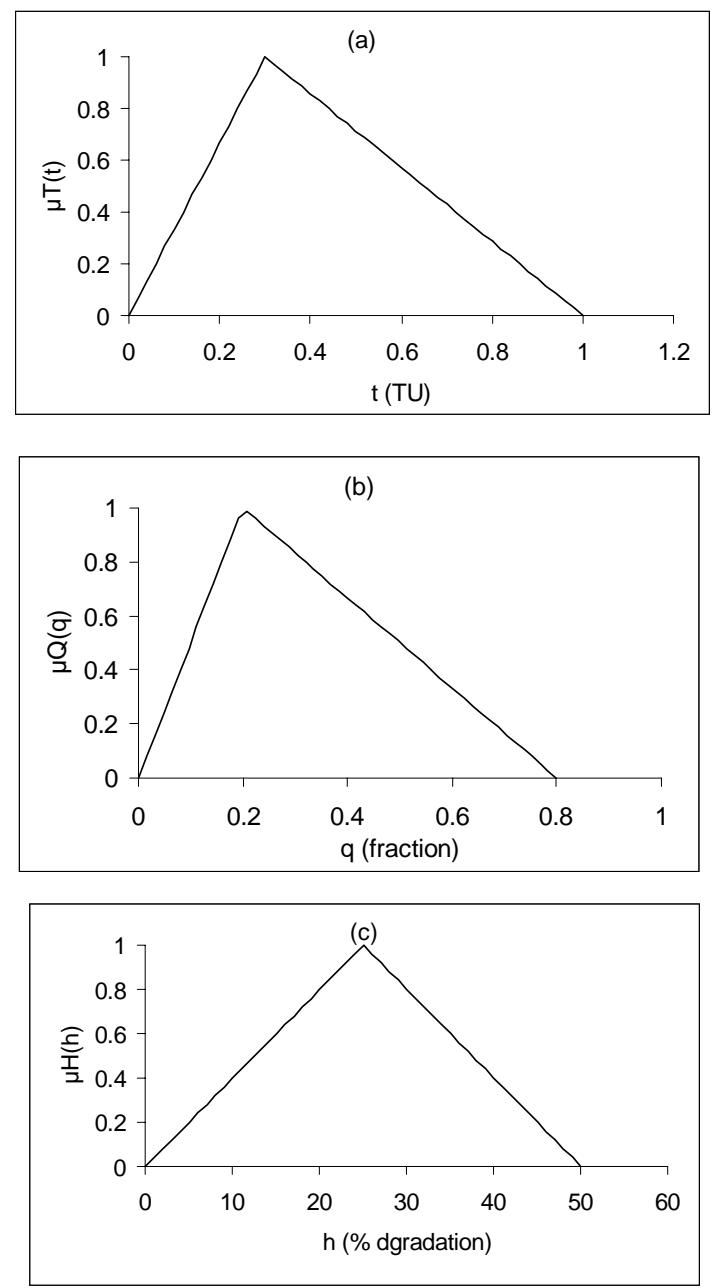

Figure 6

The stressor possibility distributions for (a) toxics-, (b) flow- and (c) habitat-related stress (expressed as $\mu_{\mathrm{x}}(\mathrm{x})$ ) derived from the descriptive data for Scenario 3 in Table 2

extensive consideration. Any procedure for deriving the fuzzification parameters would have to take cognisance of:

- correspondence between observed system assessments and the concern and risk classes projections, and

- the risk perceptions of the user community.

The former problem can probably best be addressed by analysis of a database containing both bio-monitoring and stressor data by a tool such as neural networks. The assumption is that the concern and dread levels will generally be reflected in the trends in stream bio-integrity. The latter problem is similar to the domain of risk communication except that risk values are usually in probabilistic rather than possibilistic terms.

The concern and dread assessments are also significantly affected by the SRRs. The use of fuzzy mapping as SRRs addresses this problem to some extent. With reference to SRRs it is noted that:

- If the uncertainty in the different SRRs differ widely, it is apparent that the higher uncertainty will dominate the assessment uncertainty. It may, for example, be unnecessary to insist on high confidence toxicity response data (simply because it can
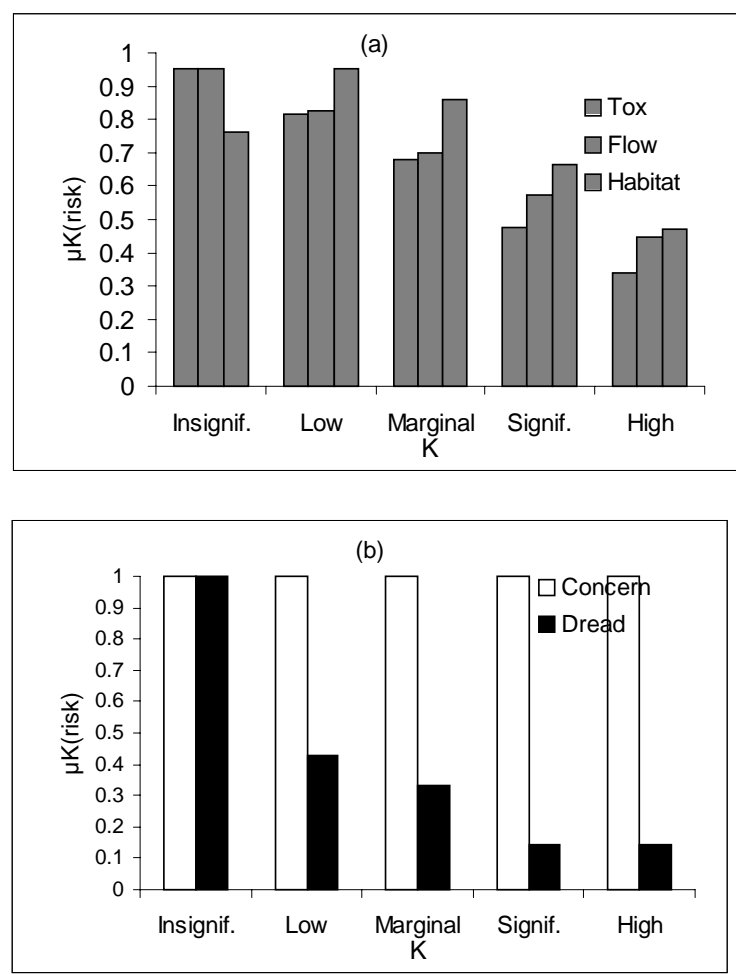

Figure 7

The classification in terms of class $\mathrm{K}$ (Fig. 1) of (a) stressorspecific effect possibility (Poss(E)) and (b) concern and dread for Scenario 3

be achieved), while having to accept very coarse data on habitat related stressor-response information.

- It has tacitly been assumed that the identification of a stressor had taken into consideration a temporal component if at all applicable. It is known that toxic substances may accumulate over a period to toxic levels in an organism (e.g. Mancini, 1983; Legierse, et al., 1999). However, for toxic substances intraorganismal stressor exposure was assumed to be proportional to the stressor magnitude, while the temporal characteristics of the stressor had been neglected.

- In the case of flow stress, the assumption that stress is simply proportional to reduction from expected flow, is probably too simplistic. It is known that a certain amount of flow variability is both normal and necessary for the functioning of most South African aquatic ecosystems (King and Louw, 1998). A more realistic description of flow-related stress would likely involve a stochastic variable whereby the range becomes abnormal.

- The duration of stress has not been explicitly addressed for any of the stressors. This paper does not particularly concern itself with the detail of such a description, except to postulate that such a descriptor will have a magnitude component and a temporal duration component, both of which could be variable. It is possible that the variables used to characterise the stress descriptor would be crisp, but the advantage of the fuzzy approach is that they could be vague or fuzzy (depending on the state of knowledge) without invalidating the approach.

Considering Eqs.(2), (3) and (5) or (6), it is trivial to recognise that there are theoretically an infinite number of stressor-specific fuzzy risk combinations that result in the same concern (or dread) value. If only a single stressor was being addressed, it would simply 

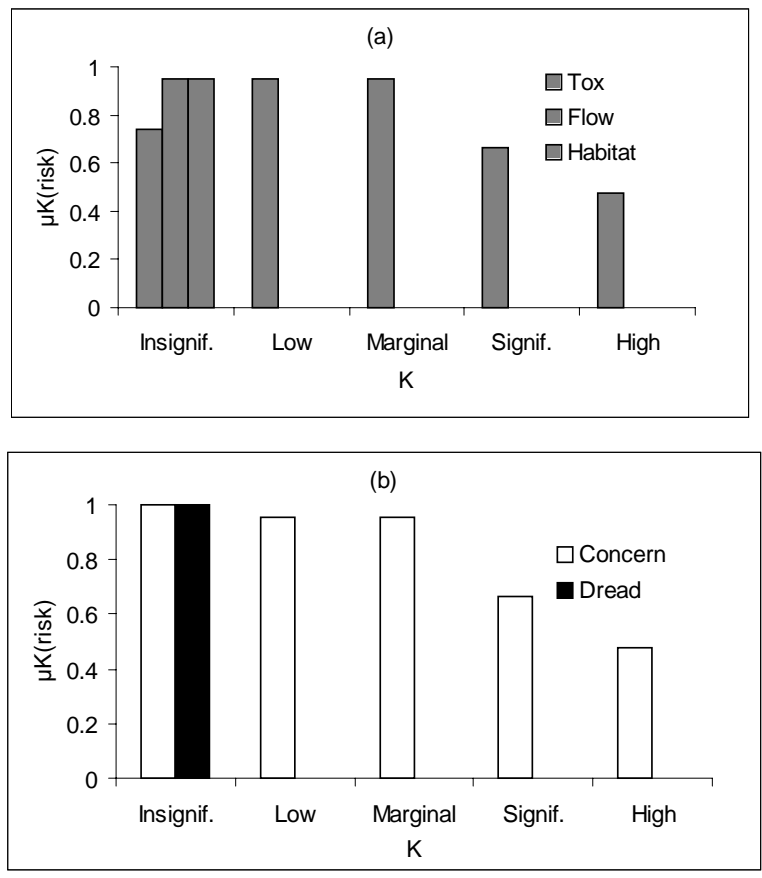

Figure 8

The classification in terms of class K (Fig. 1) of (a) stressorspecific effect possibility (Poss(E)) and (b) concern and dread for Scenario 4

require a waste load allocation-type of calculation to distribute the stressor load among stressor sources (USEPA, 1991). However, the essence of the concern and dread calculation is the aggregtion of the diverse stressors into one measure. This means that in order to select among the infinite number of source-specific stressorlevel combinations, some form of optimisation procedure would be called for. While this is a more complex task than a waste load allocation (USEPA, 1991) it also increases the management flexibility by opening the way for cost-risk-benefit calculation. This aspect requires some investigative work, although there is a substantial volume of work in the field of fuzzy optimisation (Dubois and Prade, 1994, Klir and Yuan, 1995, Sasikumar and Mujumdar, 1997).

The mathematical structure of the model is unaffected by the number of premises and propositions since it is based mostly on max and min operations. The extension to additional interactions is trivial. However, the possibility and necessity measures for the rules need to be stated as they determine the confidence in the overall assessment and this holds true for the stressor-effect implications.

\section{Conclusions}

This paper is an attempt to motivate the use of a possibilistic approach to ecological risk assessment rather than the more common probablistic approach in cases where there is epistemic uncertainty as well as stochasticity in the system being assessed. The use of fuzzy logic and a possibilistic approach to ecological risk makes use of three types of information:

- an assessment of the relationship between stressors magnitude and the expected response at a suitable level of organisation in the form of a fuzzy implication relationship,
- a possibility distribution for each stressor, and

- a logical inference model connecting direct stressor effects and the higher level end-points for the assessment in the form of a rule base.

The possibilitic ERA formulation has the advantage that it could make use of the vague information that is often all that is available for ecosystems effects, but it can also be used where precise information is available. For an application where there is no need for more precise or numeric risk data, this fuzzy set approach may be sufficient. However, the use of fuzzy variables cannot be used as a cover for bad or misleading data. The scientific quality of data is a separate issue from fuzziness. While high quality data can be fuzzified, doubtful, vague or conflicting data cannot be improved by this technique. It is necessary to be explicit with the uncertainty and vagueness in the formulation of the ecological risk assessment problem.

The parameters used in the fuzzification of data need to be considered carefully. These must be agreeable to both the risk assessor and the risk manager. This is particularly crucial where stressor response curves are very steep, i.e. where large changes in response (or fuzzy set) correspond to relatively small changes in stressor exposure.

\section{References}

BRODERIUS S and KAHL M (1985) Acute toxicity of organic chemical mixtures to the fathead minnow. Aquat. Toxic. 6 307-322.

CAIRNS J (1977) Quantification of biological integrity. In: Ballantine RK and Guarraia LJ (eds.) The Integrity of Water. United States Environmental Protection Agency, Office of Water and Hazardous Materials, Washington DC. 171-187.

CALAMARID and VIGHIM (1992) A proposal to define quality objectives for aquatic life for mixtures of chemical substances. Chemosphere $\mathbf{2 5}$ (4) $531-542$.

CASWELL H (1989) Matrix Population Models: Construction, Analysis and Interpretation. Sinauer Associates, Inc. Sunderland, Mass.

COOKE RM (1991) Experts in Uncertainty: Opinion and Subjective Probability in Science. Oxford University Press

COSTANZA RM, KEMPT WM and BOYNTON WR (1993) Predictability, scale and biodiversity in coastal and estuarine ecosystems: Implications for management. AMBIO 22 88-96.

DE LEO G and LEVIN S (1997) The multifaceted aspects of ecosystem integrity. Conser. Ecol. 1 (1) (www.consecol.org./vol1/iss1/art3). $15 \mathrm{pp}$.

DUBOIS D and PRADE H (1988) Possibility Theory: An Approach to the Computerized Processing of Uncertainty. Plenum Press, New York. $263 \mathrm{pp}$.

DUBOIS N and PRADE H(1994) Decision-making under fuzzy constraints and fuzzy criteria - Mathematical programming vs. rule-based system approach. In: Delgado M, Kacprzyk V, Verdegay J-L and Vila MA (eds.) Fuzzy Optimization: Recent Advances. Physica Verlag.

FREY HC (1993) Separating Variability and Uncertainty in Exposure Assessment: Motivations and Method. Paper93-RA-116A.02 presented at the Air \& Waste Manage. Assoc. 86 ${ }^{\text {th }}$ Ann. Meet. Denver, Colorado, June 13-18, 1993.

FREY HC and RHODES DS (1999) Quantitative Analysis of Variability and Uncertainty in Environmental Data and Models. I: Theory and Methodology Based on Bootstrap Simulation. Water Resour. and Environ. Eng. Program, North Carolina State Univ., Raleigh, NC.

HERMENS J, CANTON H, JANSSEN P and DE JONG R (1984a) QSAR's and toxicity of chemicals with anesthetic potency: acute lethal and sublethal toxicity to Daphnia. Aquat. Toxicol. 5 143-154.

HERMENS J, CANTON H, STEYGER N, WEGMAN R (1984b) Joint effects of a mixture of 14 chemicals on the mortality and inhibition of Daphnia magna. Aquat. Toxicol. 5 315-322.

JAYNES ET (1996) Probability Theory: The Logic of Science. (www.wustl.edu). 
KARR JR and DUDLEY DR (1981) Ecological perspective on water quality goals. Environ. Manage. 5 55-68.

KING J and LOUW D (1998) Instream flow assessments for regulated rivers in South Africa using the building block methodology. Aquat. Ecosyst. Health and Manage. 1 109-124.

KLIR GJ and YUAN B (1995) Fuzzy Sets and Fuzzy Logic: Theory and Applications. Prentice Hall Inc.

KRUSE R, GEBHARDT J and KLAWONN F (1994) Foundations of Fuzzy Systems. John Wiley\& Sons, Chichester, UK.

LEGIERSE KCHM, VERHAAR HJM, VAES WHJ, DE BRUIJN JHM and HERMENS JLM (1999) Analysis of the time-dependent toxicity of organophosphorus pesticides: The Critical Target Occupation model. Environ. Sci. Technol. 33 (6) 917-925.

MACKAY HM (1998) Towards a classification system for water resources in South Africa. Paper presented at the WISA Bienn. Conf., Cape Town, South Africa.

MANCINIJL (1983) A method for calculating effects, on aquatic organisms, of time varying concentrations. Water Res. 17 1355-1362.

NWA (1998) National Water Act (Act 36 of 1998) Republic of South Africa, Government Gazette. Government Printer, Pretoria, South Africa.

PEDERSON F, DAMBORG A and KRISTENSEN P (1995) Guidance Document for Risk Assessment of Industrial Waste Water. Miljøprojekt nr.28, Danish Environmental Protection Agency.
SASIKUMAR K and MUJUMDAR PP (1997) Fuzzy optimization model for water quality management of a river system. J. Water Resour. Plann. Manage. 124 (2) 79-88.

SUTER GW(II) (1993) Ecological Risk Assessment. Lewis Publishers, Boca Raton.

SUTER GW(II) (1995) Introduction to ecological risk assessment for aquatic toxic effects. In: Rand GM (ed.) Fundamentals of Aquatic Toxicology, Effects, Environmental Fate and Risk Assessment. Taylor \& Francis

TONKES M and BALTUS CAM (1997) Praktijkonderzoek aan complexe efflenetenmet de Totaal Effluent Milieubezwaarlikheid (TEM)metodiek. RIZA-rapportnummer 97.033. RIZA, Lelystad, The Netherlands.

USEPA (1991) Technical Support Document for Water Quality-based Toxics Control. Office of Water, Washington , DC. EPA/505/2-90001

USEPA (1996) Proposed Guidelines for Ecological Risk Assessment, EPA/630/R-95/002B, Risk Assessment Forum, U.S. Environmental Protection Agency, Washington, DC.

ZADEH L (1973) Outline of a new approach to the analysis of complex systems and decision processes. IEEE Trans. Syst. Man. Cybern. 3 $28-44$.

\section{Appendix}

The stressor response relationship for each stressor is delineated by a fuzzy mapping (See Figs. 2 to 4) such that:

$$
y_{1}=\left\{\begin{array}{ll}
0 & \text { if } x \leq x_{11} \\
\frac{x-x_{11}}{x_{12}-x_{11}} & \text { if } x_{11}<x<x_{12} \\
1 & \text { if } x \geq x_{12}
\end{array} \quad, \quad y_{2}=\left\{\begin{array}{ll}
0 & \text { if } x \leq x_{21} \\
\frac{x-x_{21}}{x_{22}-x_{21}} & \text { if } x_{21}<x<x_{22} \\
1 & \text { if } x \geq x_{22}
\end{array} \text { and } y_{m}=\frac{y_{1}+y_{2}}{2}\right.\right.
$$

so that $\forall y \in\left[y_{2}, y_{1}\right] \operatorname{Poss}(E)= \begin{cases}0 & \text { if } y \notin\left[y_{1}, y_{2}\right] \\ \frac{y-y_{2}}{y_{m}-y_{2}} & \text { if } y_{2} \leq y \leq y_{m} \\ \frac{y_{1}-y}{y_{1}-y_{m}} & \text { if } y_{m}<y \leq y_{m}\end{cases}$

where $y$ is the possibility distribution of the effect $\mathrm{E}$ derived from the mapping. The membership of y to class $\mathrm{L}$, $\mu \mathrm{L}(\mathrm{y})$, where class $\mathrm{L}$ is described by a trapezoidal function such that:

$$
\mu_{L}(y)= \begin{cases}0 & \text { if } y<a \text { or } y>d \\ \frac{y-a}{b-a} & \text { if } a \leq y \leq b \\ \frac{d-y}{d-c} & \text { if } c \leq y \leq d \\ 1 & \text { if } b<y<c\end{cases}
$$

